\title{
Impacto de las habilidades de comprensión lectora en el aprendizaje escolar: Un estudio realizado en una comuna de la región metropolitana, Chile
}

\author{
Impact of reading comprehension skills on school learning: $A$ study made in a commune of \\ the Metropolitan region, Chile
}

\section{Impacto das habilidades de compreensão de leitura na aprendizagem escolar: um estudo realizado em uma comunidade na região metropolitana do Chile}
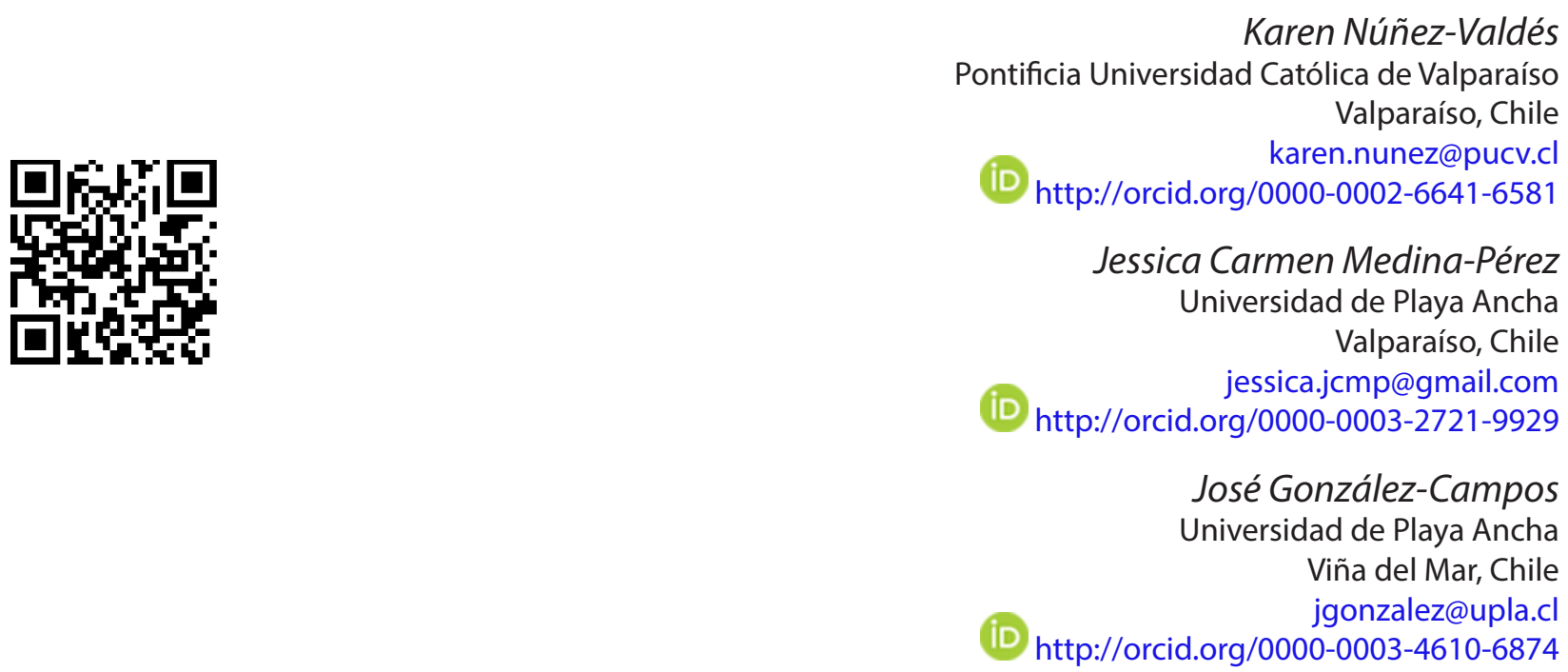

Recibido • Received • Recebido: 30 / 06 / 2017

Corregido • Revised • Revisado: 20 / 09 / 2018

Aceptado • Accepted • Aprovado: 30/ $01 / 2019$

\begin{abstract}
Resumen: La adquisición de habilidades para la comprensión lectora resulta esencial en la sociedad actual, pues no es solo una forma de aprender nuevos contenidos, sino que además es un elemento necesario para insertarse en la vida en sociedad. En este artículo se propone indagar en el impacto que poseen las habilidades de comprensión lectora en los resultados de aprendizaje de estudiantes de quinto básico de diez colegios de una comuna de la región metropolitana, Chile. La metodología a utilizar es de tipo cuantitativa, se analizan los datos a través de estadísticos descriptivos, correlaciones y jerarquizaciones. Se pretende, al concluir, conocer las habilidades que mayor impacto tienen en los resultados obtenidos por el estudiantado en las pruebas antes señaladas $y$, con ello, entregar directrices para el fortalecimiento de las habilidades de comprensión lectora más descendidas.
\end{abstract}

Palabras claves: Habilidades; comprensión lectora; evaluación estandarizada; recomendaciones para el mejoramiento de habilidades; resultados de aprendizaje. 
doi: http://dx.doi.org/10.15359/ree.23-2.2

URL: http://www.una.ac.cr/educare

CORREO: educare@una.cr

\begin{abstract}
The acquisition of skills for reading comprehension is essential in today's society, because it is not only a way of learning new contents, but also a necessary element to be inserted in life in society. In this article, we propose to investigate the impact of reading comprehension skills on the learning outcomes of fifth grade students from ten schools in a commune of the Metropolitan Region, Chile. A quantitative methodology was used. Data were analyzed through descriptive statistics, correlations, and hierarchies. It is intended to get a conclusion and to know the skills that have the greatest impact on the results obtained by the students in the tests indicated above and with that, to give guidelines for the strengthening of more realistic reading comprehension abilities.
\end{abstract}

Keywords: Skills; reading comprehension; standardized assessment; recommendations for improving skills; learning outcomes.

Resumo: A aquisição de competências para a compreensão leitora é essencial na sociedade atual, pois não é apenas uma maneira de aprender novos conteúdos, mas é também um elemento necessário para inserir na vida em sociedade. Neste artigo, propõe-se investigar o impacto que as habilidades de compreensão de leitura têm sobre os resultados de aprendizagem de alunos da quinta série de dez colégios, em uma comunidade na região metropolitana do Chile. A metodologia a ser utilizada é quantitativa, os dados são analisados por meio de estatística descritiva, correlações e hierarquias. Pretende-se, no final, conhecer as habilidades que mais causam impacto nos resultados obtidos pelos alunos nos testes citados e, com isso, fornecer orientações para o fortalecimento das habilidades de compreensão de leitura mais fluidas.

Palavras-chave: Habilidades; compreensão de leitura; avaliação padronizada; recomendações para aprimoramento de habilidades; resultados de aprendizagem.

\title{
Introducción
}

En las últimas décadas, ha tomado relevancia el incremento del capital humano, pues en general se reconoce la necesidad de su fortalecimiento como uno de los medios para lograr altas tasas de empleo, crecimiento económico, progreso social y avance en todas sus dimensiones. La Organización para la Cooperación y el Desarrollo Económico (OCDE, 2009) señala que, a raíz de esta situación, se han implementado e invertido recursos en diferentes áreas de la educación, lo que sin duda ha sido una contribución para la equidad; no obstante, aún la educación chilena muestra grandes falencias en lo que se refiere a su calidad. En el año 2011, un estudio de la Universidad de Chile dio cuenta de la preocupación e interés del Estado por los bajos resultados que se habían obtenido en pruebas estandarizadas, específicamente en el área de comprensión lectora. Ejemplo de ello, es el resultado de la prueba internacional PISA que advierte las dificultades del estudiantado chileno para relacionarse con exigencias reales de lectura, es decir, para desempeñarse como sujetos lectores competentes y autónomos en distintos contextos de lectura propios de la vida cotidiana. 
Si bien Chile es el país que más ha avanzado, en términos de resultados (puntajes) al compararlo con otros países miembros de la OCDE, el promedio del alumnado chileno es menor al puntaje promedio del estudiantado de los países miembros de esta (Consejo Nacional de la Cultura y las Artes y Centro de Microdatos, Universidad de Chile, 2011). Schiefelbein (2008) manifiesta que el desarrollo de las habilidades de comprensión lectora resultan fundamentales, ya que estas son la base y a la vez proporcionales al desempeño en otras asignaturas y, más importante aún, son habilidades para la vida. En consecuencia, la comprensión de lo que se lee es esencial para insertarse de manera adecuada en la sociedad del conocimiento, donde cada vez las personas poseen mayores exigencias a la hora de vivir en sociedad, como seleccionar y comparar información relevante, evaluar diferentes fuentes de información, entre otras. Dada la importancia de las habilidades lectoras, en este estudio se analizarán, descriptiva y asociativamente, estas variables con los resultados de la prueba estandarizada SIMCE (Matemática y Lenguaje y Comunicación) y evaluaciones estandarizadas aplicadas en el aula. Se espera tras este análisis determinar el poder predictivo de las habilidades de comprensión lectora, además jerarquizar estas según su poder predictivo en las asignaturas de Matemática, Historia, Geografía y Ciencias Sociales y Ciencias Naturales, para finalmente determinar la influencia de la variable socioeconómica en los resultados de las habilidades de comprensión lectora en la población estudiada.

\section{Formulación y fundamentación del problema de investigación}

Según el Ministerio de Educación (MINEDUC, 2011), hoy la educación ya no se centra en la recopilación y memorización de información, sino que incluye habilidades, tales como, encontrar y utilizar esta para diferentes tareas. Asimismo, se señala que la capacidad para acceder, comprender y reflexionar sobre cualquier tipo de información es esencial para que las personas puedan participar en la sociedad, que hoy se basa en el conocimiento. Holloway (1999) señala que las habilidades de comprensión lectoras son primordiales en el rendimiento académico del estudiantado. Para Olson (1994) por su parte, esto ocurre porque la habilidad de comprensión lectora otorga acceso a otros conocimientos, repercutiendo en la cognición y en los procesos de pensamiento. Cunningham y Stanovich (1997) y Smith, Mikulecky, Kibby, Dreher y Dole (2011) concuerdan en que el rendimiento en lectura no es solo la base para el rendimiento en otras asignaturas, sino que también es un prerrequisito para participar con éxito en casi todos los ámbitos de la vida actual y son la base de las habilidades laborales que se necesitarán para el siguiente milenio. Dada esta situación, resulta relevante indagar en cuál o cuáles de las habilidades de comprensión lectora el estudiantado chileno posee mayores dificultades y cómo estas dificultades impactan en los resultados de aprendizaje de otras asignaturas del currículo nacional. En esta investigación se analizarán estos elementos en colegios de una comuna de la región metropolitana que pertenecen socioeconómicamente a los grupos más desfavorecidos'.

${ }^{1}$ La OCDE (2009) coloca de manifiesto que los GSE (grupos socioeconómicos) más bajos en Chile poseen más dificultades para progresar en términos de movilidad, pues la pobreza es un círculo y la equidad una utopía. 
doi: http://dx.doi.org/10.15359/ree.23-2.2

URL: http://www.una.ac.cr/educare

CORREO: educare@una.cr

\section{Antecedentes de la prueba estandarizada en Chile: el SIMCE}

EI SIMCE es el Sistema Nacional de Evaluación de Resultados de los Aprendizajes del Ministerio de Educación de Chile. Con esta prueba se espera conocer el nivel en que se encuentra el estudiantado respecto a lo establecido en el currículo nacional y con la información obtenida tomar decisiones que favorezcan la calidad y equidad de la educación (Agencia de Calidad de la Educación, 2016). A nivel nacional, desde la década de los sesenta se intentó implementar evaluaciones que midieran los logros de aprendizaje del estudiantado chileno, así en 1968 se aplicó la primera prueba nacional, en 1982 se instauró el Programa de Evaluación del Rendimiento Escolar (PER), el que fue sustituido en 1985 por el Sistema de Evaluación de la Calidad de la Educación (SECE). Como consecuencia del contexto político imperante en 1985, surge el SIMCE, sistema de medición que tiene sus orígenes en la transformación del Estado chileno, que progresivamente abandonó su rol docente para convertirse en un Estado subsidiario, que dejó en manos de los municipios y de las instancias privadas la educación nacional (Núñez, 2014). El nuevo rol asumido por el Estado chileno requirió de un sistema de evaluación que le permitiera obtener información sobre la calidad de la educación que se comenzaba a impartir y que era financiada vía subsidios por el Estado chileno. Esta necesidad es, para Ramírez (2010), coherente con el contexto internacional, pues los Estados comenzaron a dejar su papel de proveedores directos de servicios (educación, salud, seguridad social) para convertirse en fiscalizadores de los recursos entregados a otros.

En educación el SIMCE se transformó en una herramienta capaz de entregar información del rendimiento del estudiantado en distintos niveles y asignaturas del currículo nacional al Estado chileno, por lo que la realización de esta prueba estandarizada es anual. Adicionalmente a la evaluación del currículo, se recoge información complementaria, tal como los indicadores de desarrollo personal y social (Agencia de Calidad de la Educación, 2016).

Los resultados de las pruebas SIMCE se conocen al año siguiente de su aplicación, a través de la entrega de informes por parte del Ministerio de Educación. Estos se caracterizan por otorgar información sobre las áreas evaluadas, los resultados de cada establecimiento educacional, ejemplos de preguntas con sus respuestas y recomendaciones. Los resultados se dan a conocer de manera general, ya que no se conocen los logros individuales del estudiantado, por lo que la información presentada a las comunidades de aprendizaje del país solo permite establecer comparaciones entre las escuelas con condiciones socioeconómicas similares. El informe nacional, por su parte, pretende dar a conocer a los grupos investigadores las principales variaciones de los resultados, tomando en consideración distintas variables, como género, nivel socioeconómico y dependencia del establecimiento (Agencia de la Calidad de la Educación, 2016).

Los resultados obtenidos en los últimos años dan cuenta de que no ha existido una mejora en la calidad de la educación chilena. La OCDE (2016) señala que para el caso de Chile, la probabilidad de tener un bajo rendimiento es mayor para estudiantes de grupos

4

Karen Núñez-Valdés, Jessica Carmen Medina-Pérez y José González-Campos

Los artículos de la Revista Electrónica Educare del Centro de Investigación y Docencia en Educación de la Universidad Nacional, Costa Rica, se comparten bajo términos de la Licencia Creative Commons: Reconocimiento, № Comercial, Sin Obra Derivada 3.0 Costa Rica. Las autorizaciones adicionales a las aquí delimitadas se pueden obtener en el correo: educare@una.cr 
socioeconómicos desfavorecidos, mujeres, estudiantes que asisten a escuelas en zonas rurales, que para quienes no han recibido educación parvularia y para quienes han repetido algún curso, por lo cual es indispensable, para ello, según la OCDE (2016), mejorar el acceso a una educación temprana, crear un entorno de aprendizaje en las escuelas que sea exigente y ofrezca apoyo al estudiantado, y promover la diversidad social al interior de las escuelas.

\section{Marco teórico-conceptual}

\section{Los grupos socioeconómicos y su utilización en educación}

La Agencia de Calidad de la Educación (2012) señala que el análisis de los resultados de la prueba estandarizada SIMCE se realiza conforme a diversos indicadores, internos como el desempeño del profesorado o cualquier variable que el colegio pueda controlar, y externos como la condición socioeconómica de padres, madres o estudiantes, que no pueden ser alteradas por los establecimientos educacionales. Por lo anterior, se utiliza como medida de comparación los grupos socioeconómicos (GSE), cuyo propósito es realizar una comparación más equitativa de los resultados de diferentes establecimientos. Los GSE se construyen mediante la técnica estadística de análisis de conglomerados o clusters. Este análisis permite realizar observaciones de grupos similares, que en este caso se relacionan con variables socioeconómicas. La Agencia de Calidad de la Educación (2012) establece cinco GSE, a saber, bajo (A), medio-bajo (B), medio (C), medioalto (D) y alto (E). Zamora (2011), en su análisis de los grupos socioeconómicos, advierte que la mayor movilidad se encuentra en el centro, vale decir, en los colegios que atienden a los GSE medio alto, medio y medio bajo (10,8\%; $10,1 \%$ y $10,2 \%$ en enseñanza básica). Por tanto, la menor movilidad estaría en los grupos extremos, vale decir, en el GSE alto y bajo, siendo este último caso una suerte de destino irreversible (Perticara y Sanclemente, citados por Zamora, 2011), donde es difícil de producir un movimiento. Gómez (2008) señala la importancia de desarrollar habilidades de comprensión lectora en los primeros años de educación básica, ya que son la base de una experiencia futura exitosa. Sin embargo, estudios recientes sostienen que el desempeño del estudiantado está estrechamente relacionado con los entornos socioeconómicos de sus familias y son los grupos más desfavorecidos donde se identifican estudiantes que no consiguen los prerrequisitos y habilidades base para lograr una lectura competente.

Esto es el resultado de la inequidad educativa que perpetúa otras inequidades, pues la lectura es un vehículo que permite el acceso a una gran cantidad de conocimiento, tanto actual e inmediato como el que se encuentra distante en tiempo o espacio. Además, a través de la lectura se adquiere un vocabulario que es imposible de obtener mediante el lenguaje oral; pero, sobre todo, la lectura permite, a quien la domina, aprender de manera independiente, potencializando así su desarrollo. (Gómez, 2008, p. 96) 
doi: http://dx.doi.org/10.15359/ree.23-2.2

URL: http://www.una.ac.cr/educare

CORREO: educare@una.cr

En este sentido, al pertenecer a un GSE bajo o medio bajo se está más proclive a no alcanzar el total de las habilidades de comprensión lectora, lo cual repercute directamente en el desarrollo posterior de la persona, quien debe hacer frente a estas dificultades en su vida adulta.

\section{Plan de mejoramiento educativo (PME)}

El año 2014, en el gobierno de la presidente Michelle Bachelet, el Ministerio de Educación (MINEDUC) inicia una nueva reforma educacional, que propone transformaciones en todos los ámbitos y en todos sus niveles de enseñanza, con énfasis en el fortalecimiento de la educación pública (MINEDUC, 2014). Uno de los desafíos del MINEDUC y de la Reforma del año 2014 es mejorar la calidad de la gestión pedagógica y la gestión institucional a través de los planes de mejoramiento educativo. El PME es una herramienta que ayuda a ordenar los procesos, fortalecer las gestiones positivas y superar debilidades en los establecimientos educacionales, al integrar diversos elementos característicos de cada uno de ellos, en ciclos de mejora de cuatro años, con el objetivo final de transformar al estudiantado chileno en una ciudadanía integral (MINEDUC, 2015).

En una primera fase se realiza un levantamiento estratégico basado en el Proyecto Educativo Institucional (PEI). Luego, en una segunda fase se profundiza en los períodos anuales y en el mejoramiento de las prácticas institucionales y pedagógicas. En la siguiente fase (tercera) se consolidan dichas prácticas. Finalmente, en la cuarta etapa se deben articular las prácticas institucionales y pedagógicas con el modelo de gestión escolar. Estas etapas forman parte de un ciclo de mejoramiento continuo y permanente en función de los análisis de los resultados de las evaluaciones de gestión y calidad de los aprendizajes (MINEDUC, 2013b).

La calidad de los aprendizajes es evaluada a través de pruebas internas que consideran las bases curriculares y el marco curricular vigente (conocimientos, habilidades y actitudes) en las asignaturas que se han señalado como troncales. Los instrumentos de evaluación pueden ser confeccionados por el mismo establecimiento o pueden utilizar otros que el Ministerio de Educación coloca a disposición de las instituciones educativas. Los resultados de calidad de los aprendizajes deben ser monitoreados, analizados y evaluados en periodos determinados por el establecimiento, inicio (evaluación diagnóstica), final del primer semestre (evaluación intermedia) y final del año académico (evaluación final).

En educación básica las evaluaciones de Matemática, Ciencias Naturales e Historia, Geografía y Ciencias Sociales se miden por ejes, según los requerimientos del PME, en el caso de la evaluación de Lenguaje solo se considera la comprensión lectora por ser una habilidad transversal y por el impacto que tiene en el desarrollo de las personas en aspectos cognitivos y sociales. Las habilidades y los cursos en los que se evalúan se pueden observar en la Tabla 1 y provienen de las habilidades del plan de apoyo compartido establecidas por el Ministerio de Educación, que a la vez son la referencia para la construcción de los textos escolares que utiliza el estudiantado chileno (Ministerio de Educación, 2013a).

6

Karen Núñez-Valdés, Jessica Carmen Medina-Pérez y José González-Campos

Los artículos de la Revista Electrónica Educare del Centro de Investigación y Docencia en Educación de la Universidad Nacional, Costa Rica, se comparten bajo términos de la Licencia Creative Commons: Reconocimiento, № Comercial, Sin Obra Derivada 3.0 Costa Rica. Las autorizaciones adicionales a las aquí delimitadas se pueden obtener en el correo: educare@una.cr 
doi: http://dx.doi.org/10.15359/ree.23-2.2

URL: http://www.una.ac.cr/educare

CORREO: educare@una.cr

Tabla 1: Habilidades de comprensión lectora y su evaluación según nivel

\begin{tabular}{|c|c|c|c|c|c|c|}
\hline $\begin{array}{ll} & \text { Curso } \\
\end{array}$ & $1^{\circ}$ básico & $2^{\circ}$ básico & $3^{\circ}$ básico & $4^{\circ}$ básico & $5^{\circ}$ básico & $6^{\circ}$ básico \\
\hline Desarrollo de destrezas de lectura inicial & $\mathrm{x}$ & & & & & \\
\hline Extracción de información explícita & $\mathrm{x}$ & $\mathrm{x}$ & $x$ & $\mathrm{x}$ & $\mathrm{x}$ & $\mathrm{X}$ \\
\hline Extracción de información implícita & $\mathrm{x}$ & $\mathrm{x}$ & $\mathrm{x}$ & $\mathrm{x}$ & $\mathrm{x}$ & $\mathrm{x}$ \\
\hline Reflexión sobre el contenido & & & & & $\mathrm{x}$ & $\mathrm{x}$ \\
\hline Reflexión sobre el texto & $\mathrm{x}$ & $\mathrm{x}$ & $\mathrm{x}$ & $\mathrm{x}$ & $x$ & $x$ \\
\hline $\begin{array}{l}\text { Reconocimiento de funciones gramaticales } \\
\text { y usos ortográficos }\end{array}$ & & $x$ & $x$ & $\mathrm{x}$ & $x$ & $x$ \\
\hline
\end{tabular}

Nota: Elaboración propia.

Trabajar y fortalecer las habilidades es tarea de todas las asignaturas, ya que son transversales y consideradas base para el desarrollo de habilidades superiores. Por esto, es importante conocer en profundidad cada una de ellas para identificar el nivel de desempeño del estudiantado, conocer qué son capaces de hacer y abordarlas en distintas actividades para potenciar los aspectos descendidos. El logro de las habilidades se traduce en lo que el estudiantado es capaz de hacer en:

- Extracción de información explícita: se considera la habilidad de identificar datos relevantes que se encuentran literalmente en alguna parte del texto.

- Extracción de información implícita: es la habilidad de hacer inferencias de lo leído, es decir, son aquellas interpretaciones y relaciones que se realizan de la información que no se encuentra explícita en el texto.

- Reconocimiento de funciones gramaticales y usos ortográficos: se refiere al reconocimiento y la aplicación de reglas de acentuación general, de estructuras gramaticales básicas, con énfasis en la comprensión de su función, significado de palabras según el contexto y el uso adecuado del vocabulario.

- Reflexión sobre el contenido: corresponde a relacionar el contenido de un texto con las ideas, experiencias y los conocimientos previos.

- Reflexión sobre el texto: corresponde a la habilidad previa a la lectura, la que se complementará con la comprensión total de lo leído, ya que proporciona información del texto como lo es su propósito, función o estructura (Ministerio de Educación, 2013a). 
doi: http://dx.doi.org/10.15359/ree.23-2.2

URL: http://www.una.ac.cr/educare

CORREO: educare@una.cr

\section{Comprensión lectora y sus habilidades}

Silva (2014) afirma que comprender lo que se lee va mucho más allá de una habilidad que solo se utiliza en el colegio, sino que es una herramienta que sirve de base para otras asignaturas, permite desenvolverse en el mundo de forma autónoma y efectiva, además de ayudar a formar personas más creativas y críticas. Incluso, se establece una relación directa entre la comprensión lectora y el éxito laboral. Por ello, las políticas educacionales en distintos países, especialmente miembros de la OCDE, colocan como base el desarrollo de la comprensión lectora (OCDE, 2016).

Un estudio realizado por la Universidad de Chile el 2011 (citado por Silva, 2014) entrega cifras alarmantes, donde se revela que el $84 \%$ de la población chilena no comprende de manera adecuada lo que lee. A esta información se deben agregar los resultados de comprensión lectora del SIMCE, que como se comentó anteriormente, aunque ha presentado avances, siguen siendo descendidos, por lo que es necesario invertir en el desarrollo de la comprensión de lectura. Si bien cada una de las habilidades de comprensión lectora es importante y constituye la comprensión global, las habilidades relacionadas con el vocabulario cobran mayor relevancia por ser un elemento inherente y base de la elaboración de la lengua y el progreso de diferentes competencias lingüísticas. A la vez es un indicador que se encuentra definido por variables como: la edad, nivel social, cultural y desarrollo intelectual (Hernández, 1996). Quintero (citado por Hernández, 1996) señala que una parte importante de la comprensión lectora radica en el conocimiento del significado de las palabras, por lo que, a mayor vocabulario, mayor será la comprensión.

Hart y Risley (1995) mencionan la importancia de implementar programas dirigidos al logro de la habilidad (vocabulario) lo más temprano posible, con ello se favorecerá la comprensión del estudiantado, especialmente del que se encuentra menos expuesto a una riqueza léxica. En sus estudios señalan que ya a los 5 años, el estudiantado se ha visto expuesto a una diferencia de 30 millones de palabras, dependiendo de su origen socioeconómico, lo que afectará directamente su comprensión lectora.

Otros estudios señalan que la escolarización temprana tiene un efecto positivo en los resultados de comprensión lectora (González-Betancor y López-Puig, 2015). Gutiérrez (2016) afirma que el uso de estrategias lectoras en el aula, como la lectura dialógica es efectiva para el aprendizaje del estudiantado. Y Martí y Moliner (2015) destacan que la implementación de tutorías entre pares tiene resultados positivos en el desarrollo de habilidades de comprensión lectora. Estas investigaciones dan cuenta de una serie de estrategias a utilizar en las aulas para incrementar las habilidades de comprensión lectora, por lo que la contribución de esta investigación versa sobre qué habilidades se han de fortalecer en el estudiantado. 


\section{Materiales y métodos}

Este apartado tiene por objetivo describir la metodología que se utilizará en el desarrollo de esta investigación. Se entiende, por metodología, el procedimiento que se ha escogido para llegar a conocer un determinado objeto o fenómeno, por lo que esta representa la forma de organizar el proceso investigativo, vale decir, la recolección, el procesamiento de los datos y la presentación de las conclusiones (Zorrilla, 1998). El diseño metodológico a utilizar es de tipo cuantitativo, donde se analizarán, descriptiva y correlacionalmente, los puntajes de evaluaciones estandarizadas aplicadas en el aula como a nivel nacional (SIMCE) y su relación con los puntajes obtenidos por el estudiantado en las habilidades de comprensión lectora, para finalmente aplicar algunas pruebas de significancia. Se utilizará el software r-Project, programa estadístico gratuito, usado en un lenguaje de programación $S$ y se ha utilizado ampliamente en investigaciones con enfoque estadístico. A continuación, se presenta en detalle la metodología a utilizar.

\section{Preguntas, hipótesis y objetivos de investigación}

Considerando todo lo anterior, la investigación incorpora la relación entre preguntas e hipótesis de trabajo, las que pueden observarse en la Tabla 2.

Tabla 2: Hipótesis de investigación

\begin{tabular}{|c|c|}
\hline Preguntas & Hipótesis de trabajo (o investigación) \\
\hline $\begin{array}{l}\text { ¿Cuál habilidad de comprensión lectora tiene } \\
\text { mayor poder predictivo sobre el puntaje } \\
\text { SIMCE de Matemática y de Lenguaje? }\end{array}$ & $\begin{array}{l}\text { Hipótesis 1: } \\
\text { Las habilidades básicas de comprensión lectora extracción de } \\
\text { información explícita y reflexión sobre el texto tienen mayor poder } \\
\text { predictivo sobre los puntajes del SIMCE de Matemática y Lenguaje. }\end{array}$ \\
\hline $\begin{array}{l}\text { ¿Cuál habilidad de comprensión lectora } \\
\text { tiene mayor poder predictivo sobre el } \\
\text { puntaje de la prueba de Ciencias Naturales? }\end{array}$ & $\begin{array}{l}\text { Hipótesis 2: } \\
\text { Las habilidades de comprensión lectora reconocimiento de } \\
\text { funciones gramaticales y usos ortográficos y reflexión sobre el } \\
\text { contenido tienen mayor poder predictivo sobre el puntaje de la } \\
\text { prueba de Ciencias Naturales. }\end{array}$ \\
\hline $\begin{array}{l}\text { ¿Cuál habilidad de comprensión lectora } \\
\text { tiene mayor poder predictivo sobre el } \\
\text { puntaje de la prueba de Historia, Geografía } \\
\text { y Ciencias Sociales? }\end{array}$ & $\begin{array}{l}\text { Hipótesis 3: } \\
\text { Las habilidades de comprensión lectora reconocimiento de } \\
\text { funciones gramaticales y usos ortográficos y reflexión sobre el } \\
\text { contenido tienen mayor poder predictivo sobre el puntaje de la } \\
\text { prueba de Historia, Geografía y Ciencias Sociales. }\end{array}$ \\
\hline $\begin{array}{l}\text { ¿Cuál es el nivel jerárquico que tienen las } \\
\text { habilidades de comprensión lectora? }\end{array}$ & $\begin{array}{l}\text { Hipótesis 4: } \\
\text { Extracción de información explícita se encuentra en el primer } \\
\text { nivel jerárquico de las habilidades de comprensión lectora. }\end{array}$ \\
\hline
\end{tabular}

Nota: Elaboración propia. 
doi: http://dx.doi.org/10.15359/ree.23-2.2

URL: http://www.una.ac.cr/educare

CORREO: educare@una.cr

De las preguntas anteriores emergen los objetivos de investigación.

\section{Objetivo general:}

Analizar descriptiva y asociativamente las variables de comprensión lectora con resultados SIMCE y evaluaciones estandarizadas aplicadas en el aula".

\section{Objetivos específicos:}

- Determinar el poder predictivo de las habilidades de comprensión lectora que influyen en los resultados de pruebas estandarizadas.

- Jerarquizar las habilidades de comprensión lectora según su poder predictivo en las asignaturas de Matemática, Historia, Geografía y Ciencias Sociales y Ciencias Naturales.

- Conocer la influencia de la variable socioeconómica en los resultados de las habilidades de comprensión lectora.

\section{Identificación de variables y definición operacional}

Las variables a utilizar son:

a) Habilidades de comprensión lectora correspondiente a la evaluación intermedia de $5^{\circ}$ básico (PME), a saber, extracción de información explícita, extracción de información implícita, reconocimiento de funciones gramaticales y usos ortográficos, reflexión sobre el texto y reflexión sobre el contenido. Estas son variables de tipo cuantitativas expresadas en porcentaje de logro de razón (numérica).

b) Porcentaje de logro o resultados de las evaluaciones en las asignaturas de Historia, Geografía y Ciencias Sociales y Ciencias Naturales, correspondiente a la evaluación intermedia del estudiantado de $5^{\circ}$ básico (PME). Estas variables son de tipo cuantitativas de razón (numérica).

c) Puntajes SIMCE de Lenguaje y Matemática $4^{\circ}$ básico año 2015, que corresponden a los puntajes del estudiantado que actualmente cursa $5^{\circ}$ básico. Corresponde a una variable cuantitativa de razón (numérica).

d) GSE, corresponde a una variable de tipo cualitativa ordinal. 


\section{Tipo de investigación y alcance}

Esta investigación se enmarca en el paradigma positivista, utilizando herramientas de tipo cuantitativo con alcances descriptivos y correlacionales, de corte transversal (Hernández, Fernández y Baptista, 2014).

\section{Unidad de análisis, universo y muestra}

Para esta investigación la unidad de análisis son las habilidades de comprensión lectora. Siendo la población un grupo de colegios municipales ubicados en la ciudad de Santiago en la región metropolitana. La comuna se caracteriza porque sus habitantes pertenecen mayoritariamente a un GSE bajo y medio bajo, con una tasa de escolaridad de 9,6 años, bajo el promedio nacional que es de 10,1 (Biblioteca del Congreso Nacional de Chile, 2008). El nombre de la comuna no se mencionará en esta investigación por proteger la identidad del estudiantado y sus resultados en el logro de las distintas habilidades.

La muestra es de tipo intencionada y está en función del GSE, que en este caso es representativo de los grupos más desfavorecidos socioeconómicamente en Chile (bajo y medio bajo). En consecuencia, se seleccionaron estudiantes de $5^{\circ}$ básico, cohorte 2016, de 10 colegios que poseen esta característica. Este grupo de estudiantes rindió la prueba SIMCE el año 2015, cuando cursaba 4 º básico.

\section{Técnica de recolección de datos}

Los datos que se utilizarán en este estudio fueron obtenidos tras la aplicación de pruebas estandarizadas, correspondientes a evaluaciones intermedias efectuadas el año 2016, las cuales son parte de los PME de los colegios participantes de esta investigación. Los datos fueron facilitados por los establecimientos educacionales y los resultados del SIMCE 2015 del estudiantado de $4^{\circ}$ básico, obtenidos de la plataforma de libre acceso de la Agencia de Calidad de la Educación.

\section{Técnica de análisis de datos}

Las técnicas de análisis de datos están en función del estatus métrico de las variables especificadas. En este sentido las técnicas que se utilizaran se centran inicialmente en aspectos descriptivos, luego correlacionales y, finalmente, algunas pruebas de significancia (fundamentadas en el tamaño muestral y su representatividad). 
doi: http://dx.doi.org/10.15359/ree.23-2.2

URL: http://www.una.ac.cr/educare

CORREO: educare@una.cr

\section{Aplicación y resultados}

Tras el análisis de los datos, se presentan los resultados según las distintas pruebas estadísticas que se han aplicado:

\section{Análisis N. ${ }^{\circ} 1$ : Medias según variables}

a) Medias por variables. Habilidades de comprensión lectora en estudiantes de quinto básico, descritas en la Tabla 3:

Tabla 3: Medias en habilidades de comprensión lectora en estudiantes de quinto básico

\begin{tabular}{lc}
\hline Habilidad de comprensión lectora & Media de los puntajes obtenidos \\
\hline Extracción de información explícita & 44.35 \\
Extracción de información implícita & 40.07 \\
Reconocimiento de funciones gramaticales y usos ortográficos & $\mathbf{3 1 . 7 2}$ \\
Reflexión sobre el contenido & 44.32 \\
Reflexión sobre el texto & 43.12 \\
\hline
\end{tabular}

Nota: Elaboración propia.

Se han obtenido las medias en las habilidades de comprensión lectora del estudiantado de todos los colegios participantes en esta investigación, evidenciándose que la habilidad más descendida es el reconocimiento de funciones gramaticales y usos ortográficos, donde se ha obtenido un promedio de 31.72 puntos. Esta habilidad muestra la relación con el reconocimiento y la aplicación de reglas de acentuación general, de estructuras gramaticales básicas y el uso del vocabulario según contexto. Resulta relevante identificar esta habilidad como la más descendida, pues está directamente vinculada a comprender y resignificar lo que se lee, pues se requiere de un vocabulario amplio para lograr moverse en distintos contextos, lo que para la OCDE (2016) es fundamental para el mundo globalizado en el cual vivimos.

b) Medias por variables. Evaluaciones intermedias estandarizadas aplicadas en el aula, descritas en la Tabla 4:

Tabla 4: Medias obtenidas por estudiantes de quinto básico en evaluaciones intermedias estandarizadas

\begin{tabular}{lc}
\hline Evaluación estandarizada intermedia & Media de los puntajes obtenidos \\
\hline Asignatura Ciencias Naturales & 40.89 \\
Asignatura Historia, Geografía y Ciencias Sociales & 43.46 \\
\hline
\end{tabular}

Nota: Elaboración propia. 
El estudiantado que rindió ambas evaluaciones en sus establecimientos educacionales obtuvo puntajes similares en las asignaturas, con un margen de diferencia de 2.57 puntos. Se sospecha que la diferencia es producto de la aleatoriedad, en las secciones siguientes se harán pruebas de significancia.

c) Medias por variables. Evaluación estandarizada SIMCE 2015: Las medias se muestran en la Tabla 5:

Tabla 5: Medias obtenidas por el estudiantado en evaluación estandarizada SIMCE 2015

\begin{tabular}{lc}
\hline SIMCE 2015 & Media de los puntajes obtenidos \\
\hline Lenguaje y Comunicación & 235.61 \\
Matemática & 234.83 \\
\hline
\end{tabular}

Nota: Elaboración propia.

Al observar las medias de la prueba estandarizada SIMCE no existen grandes diferencias en los puntajes. Lenguaje y Comunicación está por sobre el puntaje de Matemática en solo 0.78 puntos.

\section{Análisis N. ${ }^{\circ}$ 2: Test de medias}

El propósito de este análisis es verificar si la diferencias entre las medias es producto del azar o si existe una causa para ello. Este test se aplicó a la variable habilidades de comprensión lectora, considerando un nivel de significancia del 5\%. Los resultados del test de medias se presentan en la Tabla 6:

Tabla 6: Test de medias y sus resultados

\begin{tabular}{|c|c|c|}
\hline Variable N..$^{\circ} 1$ & Variable N. ${ }^{\circ} 2$ & Resultado del análisis \\
\hline $\begin{array}{l}\text { Extracción de } \\
\text { información explícita (X) }\end{array}$ & $\begin{array}{l}\text { Reflexión sobre el } \\
\text { contenido }(\mathrm{Y})\end{array}$ & $\begin{array}{l}\text { Al contrastar las medias de las variables } \mathrm{XeY} \text {, se evidencia que no } \\
\text { hay diferencias significativas en estas, con un p-valor de } 0.4892\end{array}$ \\
\hline $\begin{array}{l}\text { Reflexión sobre el } \\
\text { contenido }(\mathrm{X})\end{array}$ & $\begin{array}{l}\text { Reflexión sobre el } \\
\text { texto }(\mathrm{Y})\end{array}$ & $\begin{array}{l}\text { Al contrastar las medias de las variables } \mathrm{X} \text { eY, se evidencia que no } \\
\text { hay diferencias significativas entre ellas, siendo el p-valor } 0.1512\end{array}$ \\
\hline Reflexión sobre el texto & $\begin{array}{l}\text { Extracción de } \\
\text { información implícita }\end{array}$ & $\begin{array}{l}\text { Los datos soportan evidencia para rechazar la hipótesis de } \\
\text { igualdad de medias, esto significa que la variable reflexión sobre } \\
\text { el texto es significativamente mayor que la variable extracción } \\
\text { de información implícita con un p-valor de } 0.006281\end{array}$ \\
\hline $\begin{array}{l}\text { Extracción de } \\
\text { información implícita }\end{array}$ & $\begin{array}{l}\text { Reconocimiento } \\
\text { de funciones } \\
\text { gramaticales y usos } \\
\text { ortográficos }\end{array}$ & $\begin{array}{l}\text { Los datos soportan evidencia para rechazar la hipótesis de } \\
\text { igualdad de medias, esto significa que la variable extracción } \\
\text { de información implícita es significativamente mayor que } \\
\text { la variable reconocimiento de funciones gramaticales y sus } \\
\text { ortográficos, siendo el p-valor } 0.00000104\end{array}$ \\
\hline
\end{tabular}

Nota: Elaboración propia. 
doi: http://dx.doi.org/10.15359/ree.23-2.2

URL: http://www.una.ac.cr/educare

CORREO: educare@una.cr

Lo anterior nos permite establecer que existen dos variables que sus medias son significativamente menores respecto de las otras habilidades, estás son extracción de información implícita y reconocimiento de funciones ortográficas y usos ortográficos.

\section{Análisis N. ${ }^{\circ}$ 3: Análisis correlacionales}

\section{a) Correlación puntaje Ciencias Naturales con las habilidades de comprensión lectora}

Tras el análisis correlacional (nivel de significancia del $5 \%$ ) entre las habilidades de comprensión lectora con los puntajes obtenidos por el estudiantado en la prueba intermedia de Ciencias Naturales se desprende que existen dos variables que afectan significativamente los puntajes obtenidos por estos:

- Extracción de información explícita con un p-valor de 0.00000244

- Extracción de información implícita con un p-valor de 0.0265

\section{b) Correlación puntaje de Historia, Geografía y Ciencias Sociales con las habilidades comprensión lectora}

Luego del análisis correlacional realizado entre los puntajes obtenidos por el estudiantado en la prueba intermedia de Historia, Geografía y Ciencias Sociales y las habilidades de comprensión lectora, es posible determinar que la variable que afecta significativamente el puntaje obtenido por el estudiantado en esta prueba, es la extracción de información explícita con un p-valor de 0.0374 .

\section{c) Correlación entre los resultados de la prueba estandarizada SIMCE en el área de Lenguaje y Comunicación año 2015 con las habilidades de comprensión lectora}

Elanálisis correlacional realizado a los resultados obtenidos por el estudiantado en la prueba SIMCE en el área de Lenguaje y Comunicación del año 2015 y las habilidades que componen la comprensión lectora, se evidencia la existencia de una variable que afecta significativamente el puntaje obtenido por el estudiantado en la prueba SIMCE. La variable antes mencionada es reconocimiento de funciones gramaticales y usos ortográficos ( $p$-valor $=0.00129$ ). Situación ya evidenciada en el análisis de medias, donde esta habilidad se presenta como la más descendida en el grupo estudiado.

d) Correlación entre los resultados de la prueba estandarizada SIMCE en el área de Matemática año 2015 con las habilidades de comprensión lectora

Del análisis correlacional desarrollado se desprende que la variable que afecta significativamente el puntaje obtenido por el estudiantado en la prueba estandarizada SIMCE en el área de Matemática del año 2015, es el reconocimiento de funciones gramaticales y usos ortográficos ( $p$-valor $=0.000383)$. 
En síntesis, tanto en la prueba intermedia de Ciencias Naturales como en la de Ciencias Sociales existe una variable que afecta el puntaje obtenido el estudiantado de quinto básico en ambas, a saber, extracción de información explícita, habilidad que localiza claves textuales que se encuentra en cualquier parte del texto.

En el caso del SIMCE la variable que afecta significativamente el resultado obtenido por el estudiantado tanto en el área de Lenguaje y Comunicación como en el de Matemática, año 2015, es el reconocimiento de funciones gramaticales y usos ortográficos. Esta variable está asociada a la acentuación y al vocabulario que el estudiantado posee.

\section{Análisis 4: Jerarquía de las habilidades de comprensión lectora según el tipo de evaluación estandarizada, intermedia y SIMCE}

A partir de los análisis realizados se obtuvo la influencia de cada una de las habilidades de comprensión lectora en los puntajes obtenidos por el estudiantado en las pruebas aplicadas. Las jerarquías antes mencionadas se muestran en las Figuras 1, 2, 3 y 4.

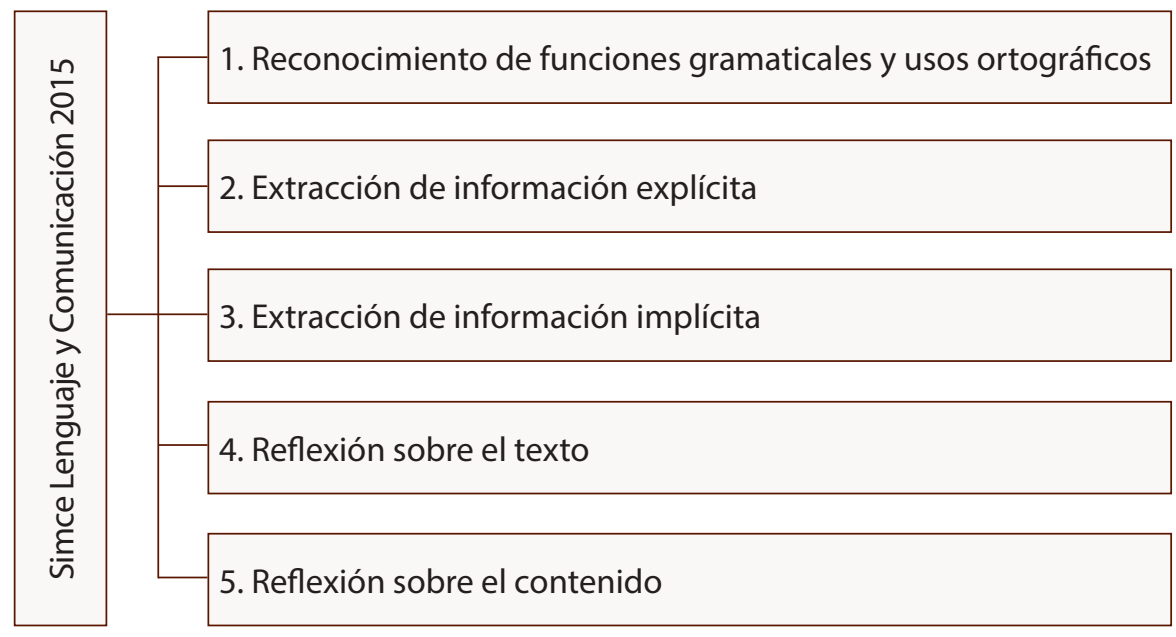

Figura 1: Jerarquía de las habilidades de comprensión lectora que influyen en los resultados SIMCE Lenguaje y Comunicación.

Nota: La figura es de elaboración propia y tiene como objetivo dar a conocer al lector las habilidades que más influyen en los resultados obtenidos en la prueba de Lenguaje y Comunicación SIMCE. 
doi: http://dx.doi.org/10.15359/ree.23-2.2

URL: http://www.una.ac.cr/educare

CORREO: educare@una.cr

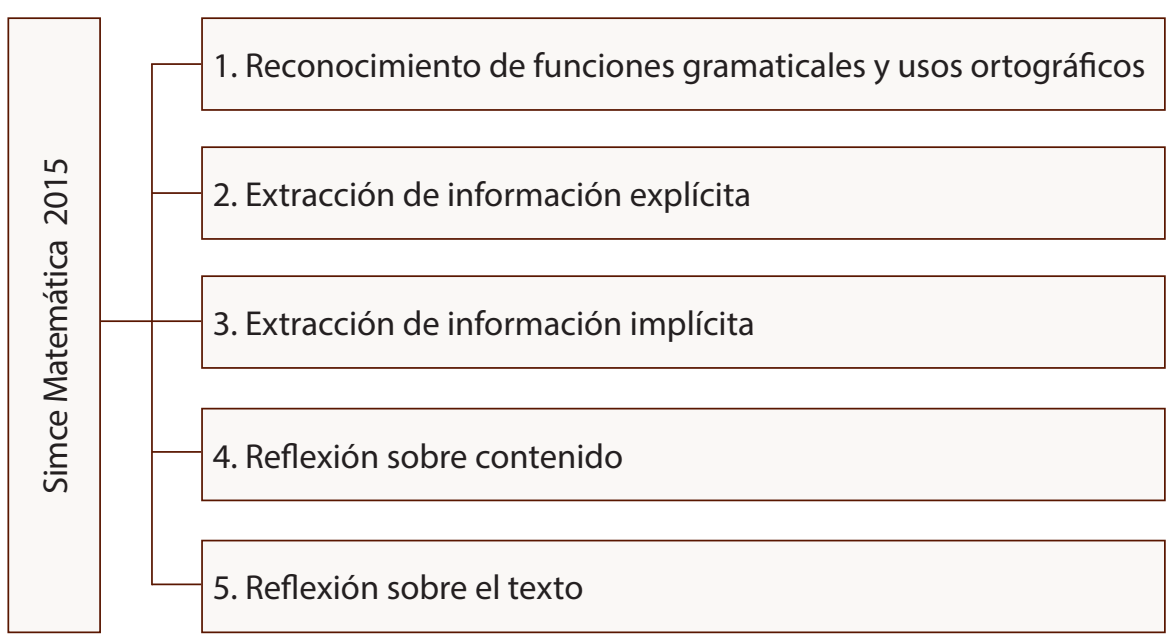

Figura 2: Jerarquía de las habilidades de comprensión lectora que influyen en los resultados SIMCE Matemática.

Nota: La figura es de elaboración propia y tiene como objetivo dar a conocer al lector las habilidades que más influyen en los resultados obtenidos en la prueba SIMCE de Matemática.

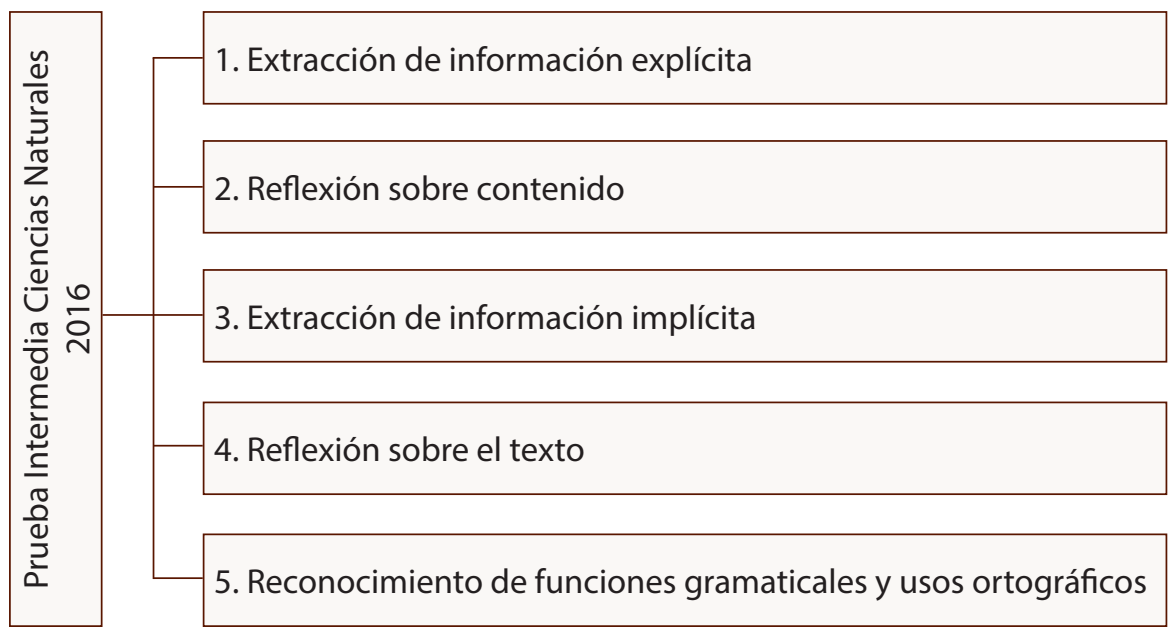

Figura 3: Jerarquía de las habilidades que influyen en el resultado de la evaluación intermedia de Ciencias Naturales.

Nota: La figura es de elaboración propia y tiene como objetivo dar a conocer al lector las habilidades que más influyen en los resultados de la evaluación intermedia de Ciencias Naturales. 


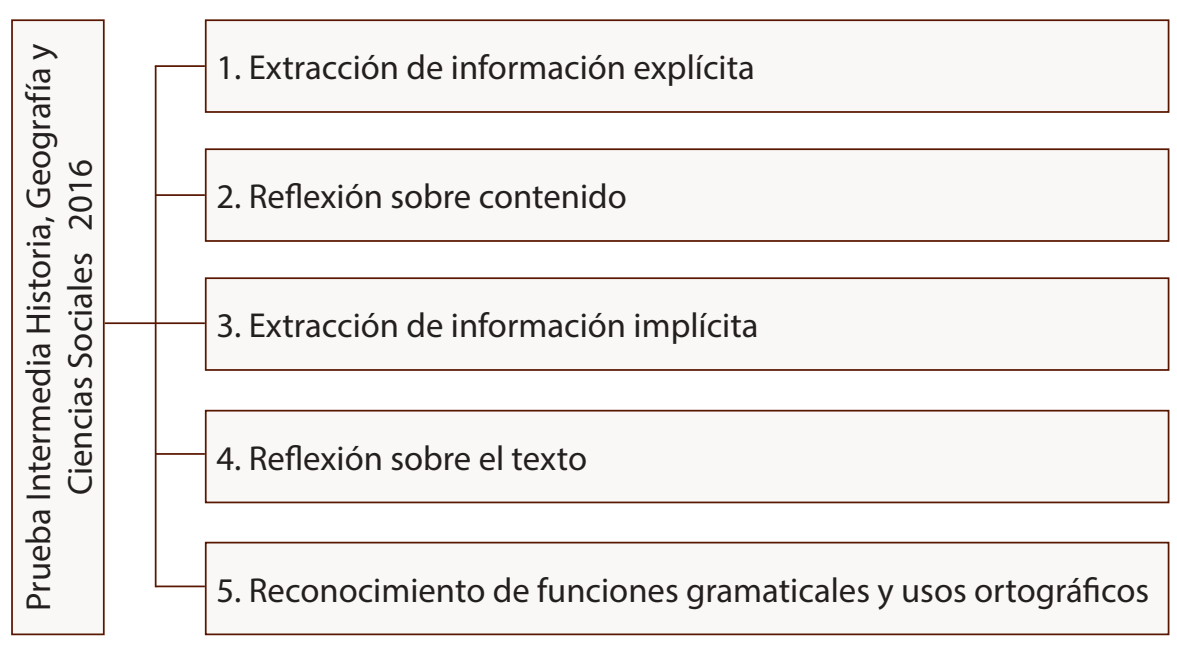

Figura 4: Jerarquía de las habilidades que influyen en el resultado de la evaluación intermedia en Historia, Geografía y Ciencias Sociales.

Nota: La figura es de elaboración propia y tiene como objetivo dar a conocer al lector las habilidades que más influyen en los resultados de la evaluación intermedia de Historia, Geografía y Ciencias Sociales.

Resulta relevante señalar que existen dos modelos de influencia en los resultados de las pruebas aplicadas al estudiantado. De una parte, los puntajes obtenidos en la prueba SIMCE están influenciados por la habilidad de reconocer las funciones gramaticales y usos ortográficos. Por otra, las evaluaciones intermedias, en términos de jerarquía, están marcados por la influencia de la habilidad de extracción de información explícita.

\section{Decisiones de las hipótesis de investigación}

Hipotesis 1: La hipótesis planteada es rechazada, a pesar de que existe dependencia significativa, superada por reconocimiento de funciones gramaticales y usos ortográficos.

Hipótesis 2: Esta hipótesis es rechazada, considerando un nivel de significancia del $5 \%$.

Hipótesis 3: Esta hipótesis es rechazada, considerando un nivel de significancia del $5 \%$.

Hipótesis 4: Esta hipótesis es rechazada, sin embargo, se debe indicar que en el 50 \% de las pruebas aplicadas la extracción de información explícita se encuentra en el primer nivel jerárquico. 
doi: http://dx.doi.org/10.15359/ree.23-2.2

URL: http://www.una.ac.cr/educare

CORREO: educare@una.cr

\section{Conclusiones y recomendaciones}

Como ya se ha mencionado, hoy vivimosen una sociedad basada en el conocimiento, donde las habilidades lectoras resultan fundamentales para el aprendizaje del estudiantado, dado que estas habilidades dan acceso a otros conocimientos, por lo cual repercuten directamente en el rendimiento en otras asignaturas del currículo nacional, pero más importante aún en la inserción de una persona en la vida en sociedad.

Al analizar descriptiva y asociativamente las variables de comprensión lectora en función de los resultados obtenidos por estudiantes de una comuna de la región metropolitana tanto en las pruebas SIMCE del año 2015 como en evaluaciones estandarizadas aplicadas en el aula durante el año 2016, otorga la oportunidad de determinar qué habilidades deben ser enfatizadas en las aulas para lograr mejores resultados $y$, en consecuencia, el aprendizaje del estudiantado en las diferentes áreas del conocimiento. Como resultado de esta investigación se ha obtenido que el reconocimiento de funciones gramaticales y usos ortográficos es la variable que más afecta el puntaje SIMCE. Lo anterior permitirá focalizar los esfuerzos en el trabajo de aula, específicamente del vocabulario desde los primeros años de vida y especialmente a lo largo de toda la etapa escolar. A mayor desarrollo de esta habilidad, mayor serán los puntajes SIMCE, no solo en Lenguaje y Comunicación, sino también en Matemática.

Según los análisis realizados resulta fundamental que el estudiantado sea capaz de reconocer y aplicar las reglas de acentuación general, de estructuras gramaticales básicas, con énfasis en la comprensión de su función y el uso del vocabulario según contexto, lo que sin duda representa un gran desafío para los GSE más bajos de nuestro país, dado que en la primera infancia ya se evidencian brechas en el vocabulario, lo cual depende de factores externos tales como el nivel educacional de los padres como el contexto en el cual se desenvuelve el niño. En este sentido, la escuela debe asumir la responsabilidad de entregar estas herramientas a sus estudiantes con el fin de disminuir la brecha existente con los grupos socioeconómicos más altos.

En términos de las habilidades que permiten mejorar tanto los puntajes como el rendimiento en asignaturas de Ciencias Naturales e Historia, Geografía y Ciencias Sociales se deben potenciar las habilidades de extracción de información explícita en los diversos tipos de textos (literarios y no literarios) que se le presenta al estudiantado, con énfasis en estrategias que apunten al desarrollo de esta habilidad, por ejemplo: destacar información relevante como personajes, lugares, entre otros, ya que el logro de ella está directamente vinculado con los resultados de estas asignaturas.

En virtud de lo analizado surgen una serie de recomendaciones en términos del fortalecimiento de las habilidades de comprensión lectora. Por un lado, es de suma relevancia trabajar estas desde los primeros años, con el fin de que el estudiantado se apropie de las estructuras gramaticales y logre desarrollar un hábito lector. De este modo la política pública 
debería enfocarse en la primera infancia, ya que es en este periodo donde es posible estimular las habilidades de comprensión lectora, especialmente en los GSE más desfavorecidos. El incorporar planes de comprensión lectora en los establecimientos educacionales desde prebásica contribuiría al aumento de palabras, de su morfología y origen, lo que es un componente indispensable en cualquier programa de aprendizaje de vocabulario. En consecuencia, resulta necesario proporcionar al estudiantado palabras afines a los contextos, con el fin de que puedan conocerlas y recordarlas en determinado momento. La enseñanza del vocabulario supone la exposición a diversos estímulos lingüísticos desde los primeros años de vida. El que se complementará con un lenguaje formal que se entregará en la escuela desde las distintas asignaturas y será potenciado con la exposición a textos diversos y de variados contenidos. Un mayor desarrollo de esta habilidad apuntará directamente a tener una alta comprensión lectora, habilidad base en las políticas educacionales de los países miembros de la OCDE, pero por sobre todo una habilidad que permite desenvolverse con propiedad en la sociedad actual, del conocimiento y la globalización.

Una vez fortalecida la habilidad de reconocimiento de funciones gramaticales y usos ortográficos y cuando el estudiantado ya haya adquirido la lecto-escritura, las políticas educativas deben focalizar sus esfuerzos en el fortalecimiento de las otras habilidades, por ejemplo, la extracción de información explícita. De acuerdo con las evidencias obtenidas, la extracción de información explícita afecta significativamente los resultados de otras pruebas como las de Ciencias Naturales y de Historia, Geografía y Ciencias Sociales.

En síntesis y de acuerdo con los resultados de esta investigación, existe una evidente influencia de la pertenencia a GSE bajos, población estudiada, pues las habilidades menos desarrolladas están vinculadas con el entorno del estudiantado, lo que supone un doble esfuerzo a nivel país, donde, en primera instancia, se debe invertir en la primera infancia, para que la población infantil adquiera habilidades vinculadas al vocabulario y, por otro lado, hacer frente a las debilidades ya existentes, como las expuestas en los resultados.

\section{Referencias}

Agencia de Calidad de la Educación. (2012). Metodología de construcción de grupos socioeconómicos. Chile: Autor. Recuperado de http://www.agenciaeducacion.cl/wpcontent/uploads/2013/02/Metodologia-de-Construccion-de-Grupos-SocioeconomicosSIMCE-2012.pdf

Agencia de Calidad de la Educación (2016). SIMCE. Chile: Autor. Recuperado de http://www. agenciaeducacion.cl/evaluaciones/que-es-el-simce/ 
doi: http://dx.doi.org/10.15359/ree.23-2.2

URL: http://www.una.ac.cr/educare

CORREO: educare@una.cr

Biblioteca del Congreso Nacional de Chile (2008). Reporte estadístico comunal. Recuperdo de https://www.bcn.cl/siit/informes/search?busqueda=la+granja\&submit=Buscar\&mindate $=+\&$ maxdate $=+$ \&pagina $=1$ \&ordenar $=$ Relevance $\&$ cantidad $=10 \& \mathrm{~K}=1$

Consejo Nacional de la Cultura y las Artes y Centro de Microdatos, Universidad de Chile. (2011). Estudio sobre el comportamiento lector a nivel nacional. Informe final 2011. Recuperado de http://plandelectura.gob.cl/recursos/estudio-sobre-comportamiento-lector-a-nivelnacional-2011/

Cunningham, A., y Stanovich, K. (1997). Early reading acquisition and its relation to reading experience and ability 10 years later. Developmental psychology, 33(6), 934-945. doi: https://doi.org/10.1037/0012-1649.33.6.934

Gómez, L. F. (2008). El desarrollo de la competencia lectora en los primeros grados de primaria. Revista Latinoamericana de Estudios Educativos, 38(3-4), 95-126. Recuperado de http:// www.redalyc.org/pdf/270/27012440005.pdf

González-Betancor, S. M. y López-Puig, A. (2015). Early schooling: Its influence on reading comprehension at primary level. Cultura y Educación, 27(2), 237-270 doi: https://doi.org/1 $\underline{0.1080 / 11356405.2015 .1034533}$

Gutiérrez, R. (2016). Efectos de la lectura dialógica en la mejora de la comprensión lectora de estudiantes de educación primaria. Revista de Psicodidáctica, 21(2), 303-320. doi: https:// doi.org/10.1387/RevPsicodidact.15017

Hart, B. y Risley, T. R. (1995). Meaningful differences in the everyday experience of young American children. Michigan: P.H. Brookes.

Hernández, A. (1996). El papel del vocabulario en la enseñanza de la comprensión lectora y composición escrita. Aula, 8, 239-260. Recuperado de https://gredos.usal.es/jspui/ bitstream/10366/69248/1/El papel del vocabulario en la ensenanza.pdf

Hernández, R., Fernández, C. y Baptista, P (2014). Metodología de la investigación. México: McGraww-Hill.

Holloway, J. H. (1999). Improving the reading skills of adolescents. Educational Leadership, 57(2), 80-81.

Martí, J. B. y Moliner, L. (2015) Una experiencia de tutoría entre iguales fija en educación primaria para la mejora de la comprensión lectora y las habilidades sociales. Revista de Investigación en Educación, 13(2), 206-218. Recuperado de https://dialnet.unirioja.es/ servlet/articulo?codigo $=5487611$ 
Ministerio de Educación (2011). PISA Evaluación de las competencias lectoras para el siglo XXI. Marco de evaluación y preguntas de la prueba. Recuperado de http://ww2.educarchile. cl/UserFiles/P0001/file/Documentos Evaluacion2012/PISA\%20Evaluaci\%C3\%B3n\%20 de\%20las\%20Competencias\%20Lectoras\%20para\%20el\%20siglo\%20X.pdf

Ministerio de Educación (2013a). Habilidades evaluadas en el Plan de Apoyo Compartido. Recuperado de http://www.siteal.iipe.unesco.org/sites/default/files/chi plan de apoyo compartido.pdf

Ministerio de Educación (2013b). Orientaciones técnicas para liderar el plan de mejoramiento educativo.Santiago, Chile:Autor. Recuperado de http://www.salgadoanoni.cl/wordpressjs/ wp-content/uploads/2015/05/orientaciones tecnicas pme.pdf

Ministerio de Educación (2014). ¿Qué es la Reforma Educacional? Recuperado de https://www. ayudamineduc.cl/ficha/que-es-la-reforma-educacional

Ministerio de Educación (2015). Orientaciones técnicas para sostenedores y directivos escolares. Plan de Mejoramiento Educativo: Nuevo enfoque a 4 años. Santiago, Chile: Autor. Recuperado de http://ww2.educarchile.cl/PORTAL.HERRAMIENTAS/mejoraescolar/contenido-web/ Apoyo-orientaciones/1-PME/2-orientaciones-tecnicas-para-sostenedores-y-directivosescolares-fase-1.pdf

Núñez, K. (2014). Retroalimentación: Una tarea pendiente del sistema de evaluación de resultados de aprendizaje (SIMCE) en Chile. Revista Electrónica Formación y Calidad Educativa, 2(1), 97-110.

Olson, D. R. (1994). The world on paper. The conceptual and cognitive implications of writing and Reading. Cambridge: Cambridge University Press.

Organización para la Cooperación y el Desarrollo Económicos (2009). La educación superior en Chile. Recuperado de http://www.opech.cl/educsuperior/politica educacion/la es en chile ocde.pdf

Organización para la Cooperación y el Desarrollo Económicos (2016). PISA: Estudiantes de bajo rendimiento. Por qué se quedan atrás y cómo ayudarles a tener éxito. Resultados principales. Recuperado de http://www.oecd.org/pisa/keyfindings/PISA-2012-Estudiantes-de-bajorendimiento.pdf

Ramírez, A. A. (2010). La evaluación como herramienta para mejorar los procesos educativos de poblaciones socialmente vulnerables. Revista Iberoamericana de Educación, 53(5), 1-10. Recuperado de rieoei.org/deloslectores/3778Ramirez.pdf 
doi: http://dx.doi.org/10.15359/ree.23-2.2

URL: http://www.una.ac.cr/educare

CORREO: educare@una.cr

Schiefelbein, E. (2008). Desafíos para los investigadores en educación de América Latina. Magis. Revista Internacional de Investigación en Educación, 1(1), 31-44. Recuperado de http:// www.redalyc.org/articulo.oa?id=281021687003

Silva, M. (2014). La comprensión lectora es una habilidad para la vida. Educarchile. Recuperado de http://www.educarchile.cl/ech/pro/app/detalle?ID=225396

Smith, M. C., Mikulecky, L., Kibby, M. W., Dreher, M. J. y Dole, J. A. (2011). What will be the demands of literacy in the workplace in the next millennium? Reading Research Quarterly, 35(3), 378383. doi: https://doi.org/10.1598/RRQ.35.3.3

Zamora, G. (2011). Movilidad escolar en Chile. Análisis de las implicancias para la calidad y equidad de la educación. Estudios Pedagógicos, 37(1), 53-69. doi: https://doi.org/10.4067/ $\underline{\text { S0718-07052011000100003 }}$

Zorrilla, S. (1998). Introducción a la metodología de la investigación. México: Editorial Cal y Arena. 